\title{
Chemokine expression of oral fibroblasts and epithelial cells in response to artificial saliva
}

\author{
Heinz-Dieter Müller ${ }^{1,2}$ • Barbara Cvikl ${ }^{1,3}$ - Adrian Lussi ${ }^{1} \cdot$ Reinhard Gruber $^{1,2,4}$
}

Received: 3 April 2015 / Accepted: 26 August 2015 / Published online: 5 September 2015

(C) Springer-Verlag Berlin Heidelberg 2015

\begin{abstract}
Objectives Artificial saliva is widely used to overcome reduced natural salivary flow. Natural saliva provokes the expression of chemokines in oral fibroblasts in vitro. However, if artificial saliva changes the expression of chemokines remains unknown.

Materials and methods Here, we investigated the ability of Saliva Orthana ${ }^{\circledR}$, Aldiamed ${ }^{\circledR}$, Glandosane ${ }^{\circledR}$, and Saliva Natura ${ }^{\circledR}$ to change the expression of chemokines in human oral fibroblasts and the human oral epithelial cell line HSC-2 by means of reverse transcription polymerase chain reaction and immunoassays. Mucins isolated from bovine submaxillary glands and recombinant human mucin 1 were included in the bioassay. Formazan formation and LIVE/DEAD ${ }^{\circledR}$ staining determined the impact of artificial saliva on cell viability. The involvement of signaling pathways was determined by pharmacologic inhibitors and Western blotting.

Results In gingival fibroblasts, Saliva Orthana ${ }^{\circledR}$-containing mucins provoked a significantly increased expression of CXC ligand 8 (CXCL8, or interleukin 8), CXCL1, and CXCL2. Immunoassays for CXCL8 and CXCL1 confirmed
\end{abstract}

Reinhard Gruber

reinhard.gruber@zmk.unibe.ch; reinhard.gruber@meduniwien.ac.at

1 Department of Preventive, Restorative and Pediatric Dentistry, School of Dental Medicine, University of Bern, Freiburgstrasse 7, 3010 Bern, Switzerland

2 Laboratory of Oral Cell Biology, School of Dental Medicine, University of Bern, Bern, Switzerland

3 Department of Conservative Dentistry and Periodontology, Medical University of Vienna, Vienna, Austria

4 Department of Oral Biology, Medical University of Vienna, Sensengasse 2a, 1090 Vienna, Austria the translation at the protein level. The respective dilution of artificial saliva had no impact on formazan formation and LIVE/DEAD ${ }^{\circledR}$ staining. Mucins isolated from bovine submaxillary glands also increased the panel of chemokine expression in gingival fibroblasts. BAY 11-7082, a nuclear factor kappa-light-chain-enhancer of activated B cells (NF-kB) inhibitor, but also TAK-242, an inhibitor of toll-like receptor 4 signaling, blocked chemokine expression of Saliva Orthana ${ }^{\circledR}$ and bovine mucins. In HSC-2 cells, Glandosane ${ }^{\circledR}$ significantly increased CXCL8 expression.

Conclusions Saliva Orthana ${ }^{\circledR}$ stimulated chemokine expression in gingival fibroblasts. Mammalian mucins, but also possible contaminations with endotoxins, might contribute to the respective changes in gene expression. Epithelial cells have a differential response to artificial saliva with Glandosane ${ }^{\circledR}$ changing CXCL8 expression.

Clinical relevance Artificial saliva can incite a cellular response, if however the changing expression of chemokines by isolated fibroblasts and epithelial cells in vitro translates into a clinical condition, is not clear.

Keywords Artificial saliva $\cdot$ Inflammation $\cdot$ Chemokines Gingival

\section{Introduction}

Saliva is a complex cocktail of compounds being produced by the submandibular and the parotid glands. Among these compounds are proteoglycans, enzymes, antibacterial compounds, and growth factors [1]. Saliva supports food digestion, oral hygiene, and prevents dental erosion $[2,3]$. Healthy people produce around 0.75 to 1.51 per day [4]. However, Sjögren syndrome, radiotherapy, medication, and aging are associated with xerostomia, also termed dry mouth disease [5]. The 
production of saliva can drop to almost zero, provoking multiple and severe symptoms such as mucositis, dysesthesia, and deep caries lesions $[6,7]$. Xerostomia necessitates the use of extra lubricants, commonly termed artificial saliva.

Artificial saliva should closely mimic the composition and the biophysical properties of natural saliva [8]. Electrolytes provide the buffering capacity and support remineralization of the enamel surface, sugar alcohols serve as thickeners and sweeteners, and aroma compounds are odorants and increase the appeal of the product [9]. Enzymes such as amylase and lipases help the digestion of starch and lipids, respectively [10]. Glycoproteins change the viscoelastic properties allowing the formation of a stable film in the oral cavity [11]. Mucins are a group of high molecular-weight glycoproteins in saliva $[12,13]$. Even though artificial saliva mimics the physiological counterpart, the composition of natural saliva is by far more complex than the substitute's [14]. The question arises what are the key components that make artificial saliva behave like natural saliva.

Natural saliva is commonly characterized by its chemical composition and viscoelastic properties [15]. Another way of characterizing natural saliva is bioassays, revealing the cellular responses. For example, natural saliva can provoke a robust increase in the expression of chemokines in oral fibroblasts, but only moderate changes in epithelial cells [16]. Chemokines are a large family of small signaling proteins with their main function to recruit cells of the immune system to an inflammatory site [17]. CXC ligand 8 (CXCL8, or interleukin 8), CXCL1, and CXCL2 are among the chemokines that are expressed by gingival fibroblasts when exposed to natural saliva [16]. The underlying intercellular signaling pathways that control the expression of the chemokines in response to whole saliva have recently been studied [16].

Inflammatory signal transduction that can be induced by inflammatory cytokines and endotoxins is carried out by intracellular nuclear factor kappa-light-chain-enhancer of activated $\mathrm{B}$ cells $(\mathrm{NF}-\mathrm{kB})$ and mitogen-activated protein (MAP) kinase including ERK, JNK, and p38 [18]. Pharmacologic inhibitors can reveal the involvement of the signaling pathways in gene expression. For example, chemokines such as CXCL8 play a crucial role in wound healing involving activation of NF-KB and MAP kinase pathways [19]. Myofibroblasts and neutrophils also require NF- $\mathrm{KB}$ and MAPK signaling for the secretion of CXCL1 [20] and CXCL2 [21], respectively. Whole saliva involves NF-KB, p38, and ERK to increase CXCL8 expression [16]. The contribution of endotoxins can be investigated with toll-like receptor inhibitors that act upstream of NF-kB signaling [22].

Here, we take advantage of an in vitro bioassay using oral fibroblasts and the oral epithelial cell line HSC-2 to evaluate the impact of various commercial preparations of artificial saliva on chemokine expression.

\section{Materials and methods}

\section{Cultivation of gingival fibroblasts and oral epithelial cells}

Human gingival fibroblasts were isolated from biopsies after informed consent was obtained (Ethics Committee of Bern, Switzerland). Gingival fibroblasts and oral epithelial cells (HSC-2 cell line; kindly provided by Prof. Rausch-Fan, Department of Periodontology, Medical University of Vienna, Vienna, Austria) were cultured in Dulbecco's Modified Eagle Medium (DMEM, Invitrogen Corporation, Carlsbad, CA) supplemented with $10 \%$ fetal calf serum (FCS; PAA Laboratories, Linz, Austria) and antibiotics (Life Technologies, Carlsbad, CA, USA) at $37{ }^{\circ} \mathrm{C}, 5 \% \mathrm{CO}_{2}$, and $95 \%$ humidity. In total, three strains of gingival fibroblasts were established and less than 10 passages were used for the experiments. For all experiments, cells were seeded at a concentration of 30,000 cells $/ \mathrm{cm}^{2}$ into culture dishes 1 day prior to stimulation.

\section{Stimulation of gingival fibroblasts and oral epithelial cells}

Gingival fibroblasts and HSC-2 cells were incubated with a 10-fold dilution of artificial saliva Orthana ${ }^{\circledR}$ (A.S. Pharma, Hampshire, UK), Aldiamed ${ }^{\circledR}$ (Certmedica International GmbH, Aschaffenburg, Germany), Glandosane ${ }^{\circledR}$ (Cell pharm, Bad Vilbel, Germany), and Saliva Natura ${ }^{\circledR}$ (Medac $\mathrm{GmbH}$, Wedel, Germany). Cells were also incubated with $50 \mu \mathrm{g} / \mathrm{ml}$ mucin from bovine submaxillary glands type I-S (Sigma-Aldrich, St. Louis, MO) or $25 \mu \mathrm{g} / \mathrm{mL}$ recombinant human mucin 1 (Hölzel Diagnostika, Cologne, Germany). For positive controls, cells were incubated with a 10-fold dilution of human sterile saliva as described recently [16]. If not otherwise indicated, cells were exposed to artificial saliva, natural saliva, and mucins for 6 and $24 \mathrm{~h}$ for immunoassays and viability tests. The metabolic activity was determined by the conversion of MTT (Sigma, St. Louis, MO) into formazan crystals. LIVE/DEAD ${ }^{\circledR}$ staining was performed with a kit from Enzo Life Sciences AG (Lausen, Switzerland).

\section{Reverse transcription polymerase chain reaction and immunoassay}

Total RNA was isolated with the High Pure RNA Isolation Kit, including digestion with 180 Units DNase I (Hoffmann-La Roche, Basel Switzerland). Reverse transcription (RT) was performed with Transcriptor Universal cDNA Master (Roche). Reverse transcription polymerase chain reaction (RT-PCR) was done with the FastStart Universal SYBR Green Master (Roche). To quantify cDNA in the samples, the 7500 RealTime PCR System (Applied Biosystems, Life Technologies, Carlsbad, CA) was used. Primer designing was done online via the Universal ProbeLibrary Assay Design Center (Roche), and sequences are provided in Table 1. Relative gene 
Table 1 Primer sequences of the investigated chemokines

\begin{tabular}{llll}
\hline Gene & Forward primer & Reverse primer & Reference \\
\hline CXCL8 & aacttctccacaaccctctg & ttggcagccttcctgattc & {$[34]$} \\
CXCL1 & tcctgcatccccatagtta & cttcaggaacagccaccagt & {$[16]$} \\
CXCL2 & cccatggttaagaaaatcatcg & cttcaggaacagccaccaat & {$[16]$} \\
CXCL3 & aaatcatcgaaaagatactgaacaag & ggtaagggcagggaccac & {$[16]$} \\
CXCL6 & gtccttcgggctccttgt & cagcacagcagagacaggac & {$[16]$} \\
CCL7 & gaaagcctctgcagcacttc & aatctgtagcagcaggtagttgaa & {$[16]$} \\
CCL20 & gctgcttgatgtcagtgct & gcagtcaagttgcttgctg & {$[16]$} \\
\hline
\end{tabular}

expression was calculated with the $2^{-\Delta \Delta \mathrm{Ct}}$ method [23]. The immunoassay for human CXCL8 and CXCL1 was obtained from R\&D Systems (Minneapolis, MN, USA).

\section{Inhibition of chemokine signal activation pathways}

Nuclear factor kappa-light-chain-enhancer of activated B cells $(\mathrm{NF}-\mathrm{kB})$ and mitogen-activated protein (MAP) kinase-related inflammatory pathways were inhibited using BAY11-7082 (Enzo Life Sciences, Inc., Farmingdale, NY), SB203580, U0126, and SP600125 (all Santa Cruz Biotechnology, Santa Cruz, CA), respectively. The inhibitors were used at $10 \mu \mathrm{M}$ for blocking NF-KB, p38, ERK, and JNK, respectively. TAK242 (Calbiochem, Merck Millipore, Darmstadt, Germany) was used at $25 \mu \mathrm{M}$ to inhibit TLR4 signaling.

\section{Western blot analysis}

Human gingival fibroblasts were cultivated with serum-free medium overnight. Cells were stimulated with a 10-fold dilution of Saliva Orthana ${ }^{\circledR}$ and $50 \mu \mathrm{g} / \mathrm{ml}$ bovine mucins for $30 \mathrm{~min}$. Whole human saliva, IL-1 (10 ng/ml), and tumor necrosis factor alpha (TNF- $\alpha, 10 \mathrm{ng} / \mathrm{ml}$ ) served as positive control. Serum-free medium was the negative control. Sodium dodecyl sulfate (SDS)
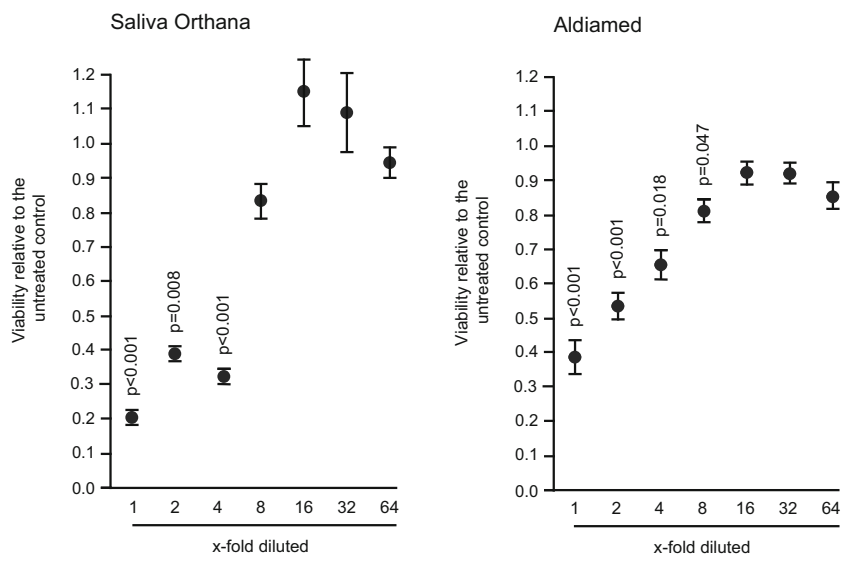

Fig. 1 Viability of gingival fibroblasts exposed to artificial saliva. Gingival fibroblasts were exposed to artificial saliva at the indicated dilutions with serum-free medium for $24 \mathrm{~h}$. Formazan formation was performed to measure cell viability. Data were normalized to the levels containing cell extracts were separated by SDS-PAGE and transferred onto nitrocellulose membranes (Whatman, GE Healthcare, General Electric Company, Fairfield, CT). Primary antibody binding was accomplished with specific phospho-NF$\mathrm{kB}$ and $\beta$-actin antibodies (Cell Signaling Technology, Danvers, MA). Quantitative secondary antibody binding was detected by near-infrared absorbing dyes with the appropriate imaging system (LI-COR Biosciences; Lincoln, NE).

\section{Statistical analysis}

Data were compared using Kruskal-Wallis and post hoc MannWhitney $U$ tests, or Friedman tests. Data with TAK-242 were evaluated with paired $t$ test. Significance was assigned at the $p<0.05$ level. Statistical analysis was performed using GraphPad Prism 6.0 (GraphPad Software Inc., San Diego, USA).

\section{Results}

\section{Viability of gingival fibroblasts exposed to artificial saliva}

To rule out a possible toxicity in the bioassay, gingival fibroblasts were exposed to various concentrations of artificial
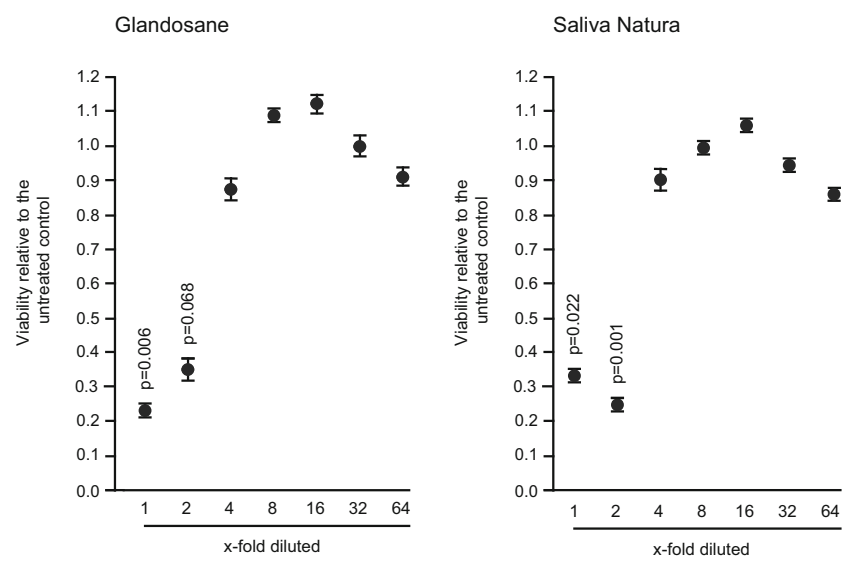

of control cultures with serum-free medium alone. Data points represent the mean \pm standard deviation of three independent experiments with two cell donors each. Not indicated are $p$ values $>0.1$ 

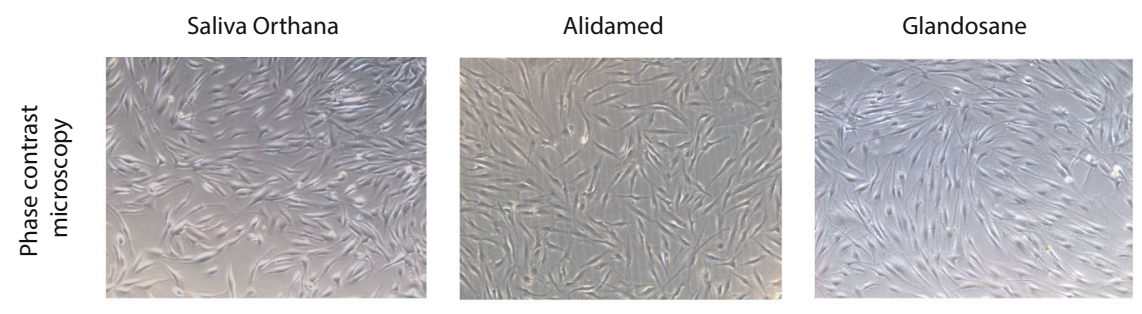

Saliva Natura
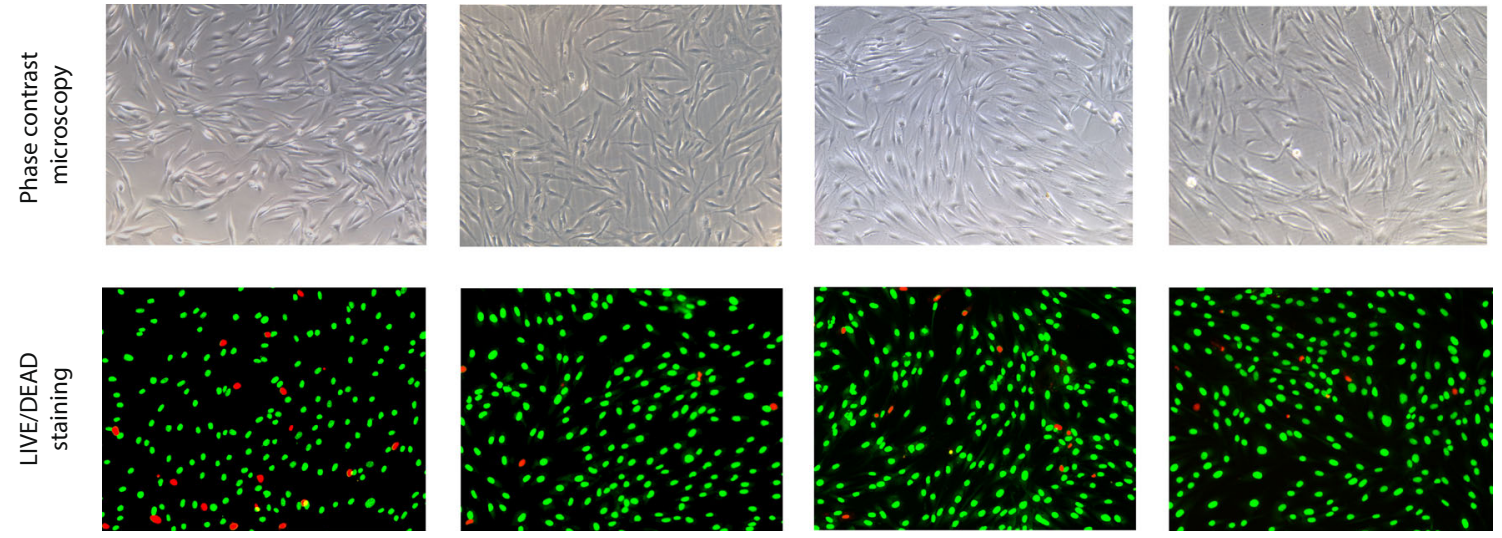

Fig. 2 Phase contrast and LIVE/DEAD ${ }^{\circledR}$ staining microscopy. Gingival fibroblasts were exposed to 10 -fold diluted artificial saliva for $24 \mathrm{~h}$. Phase contrast microscopy revealed a regular fibroblastic morphology, independent of the artificial saliva. LIVE/DEAD ${ }^{\circledR}$ staining showed that

most of the cells were green, indicating that the cells were alive (10-fold magnification). Cells were washed prior to taking pictures because Aldiamed ${ }^{\mathbb{R}}$ covers the cells with a dense precipitation layer

saliva as indicated in Fig. 1. At a 10-fold dilution, all four preparations of artificial saliva reached the control levels of formazan formation. In support of these observations, the morphology of cells and the LIVE/DEAD ${ }^{\circledR}$ staining indicated that a 10 -fold dilution of artificial saliva had no visible adverse effects (Fig. 2). Thus, further experiments were performed with a 10 -fold dilution of artificial saliva.

\section{Chemokine expression of gingival fibroblasts and oral epithelial cells exposed to artificial saliva}

Next, we determined if artificial saliva could change the expression of a panel of chemokines. Among the four preparations of artificial saliva, only Saliva Orthana ${ }^{\circledR}$, but not Aldiamed $^{\circledR}$, Glandosane ${ }^{\circledR}$, and Saliva Natura ${ }^{\circledR}$ provoked a significant increase of CXCL8, CXCL1, and CXCL2 expression in gingival fibroblasts (Fig. 3). No significant changes of the chemokines were observed with the other artificial saliva
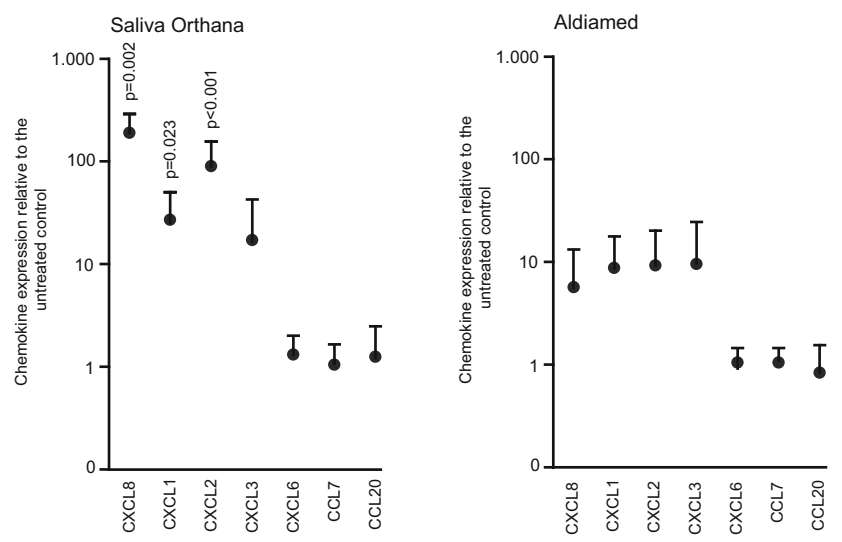

Fig. 3 Chemokine response of oral fibroblasts exposed to artificial saliva. Gingival fibroblasts were exposed to a 10 -fold diluted concentration of artificial saliva for $6 \mathrm{~h}$. A panel of chemokines was included in the RT-PCR assay. Data were normalized to expression preparations. The increased production of CXCL8 and CXCL1 in response to Saliva Orthana ${ }^{\circledR}$ was also evident at the protein level (Fig. 4). Dose- and time-response experiment showed that Saliva Orthana ${ }^{\circledR}$ increased CXCL8 expression maximally at conditions selected for the bioassay (Fig. 5). In contrast, HSC-2 cells exposed to Glandosane ${ }^{\circledR}$ significantly increased CXCL8 chemokine expression, and Aldiamed ${ }^{\circledR}$ even showed a trend toward a reduced CXCL1 expression compared to the unstimulated epithelial cells (Table 2).

\section{Mammalian mucins increased CXCL8 expression of gingival fibroblasts}

Saliva Orthana ${ }^{\circledR}$ contains mucins, in contrast to the other three preparations of artificial saliva. In line with the possible role of mucins to cause the expression of chemokines in gingival fibroblasts, mucins purified from bovine submandibular glands significantly increased CXCL8 and CXCL2, and with

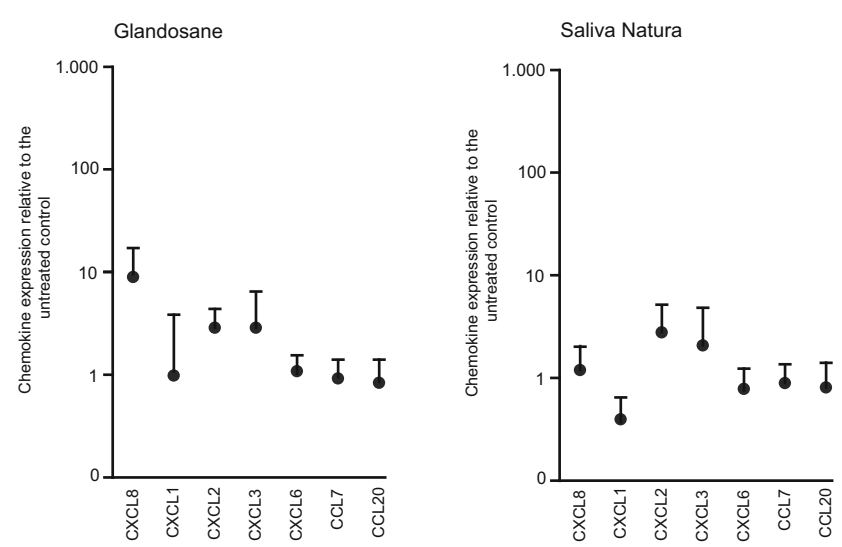

levels of control cultures with serum-free medium alone. Circles represent the mean \pm standard deviation of six experiments with two cell donors. Not indicated are $p$ values $>0.1$ 
Fig. 4 CXCL8 and CXCL1 protein production in response to artificial saliva. Gingival fibroblasts were exposed to a 10 -fold diluted concentration of artificial saliva for $24 \mathrm{~h}$. CXCL8 and CXCL1 protein production were measured with immunoassays. Data were normalized to expression levels of control cultures with serum-free medium alone. Circles represent the mean \pm standard deviation of six independent experiments. Not indicated are $p$ values $>0.1$

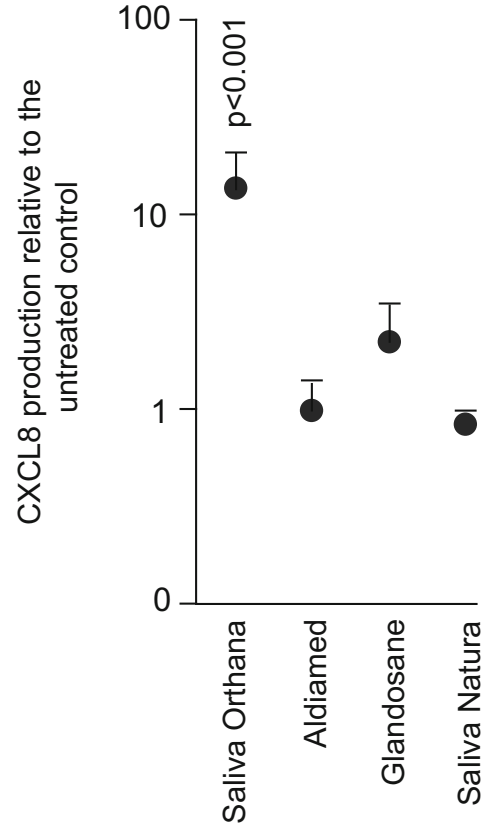

a strong trend, also CXCL1 expression. Interestingly, recombinant mucin 1 stimulated the expression of CXCL8, but not the other chemokines in gingival fibroblasts (Fig. 6).

\section{Inhibiting TLR4 and NF-KB pathways reduces gingival chemokine expression}

Gingival fibroblasts were co-cultivated with TLR4, NF-кB, and MAP kinase inhibitors and stimulated with Saliva Orthana ${ }^{\circledR}$ or bovine mucins. CXCL8, CXCL1, and CXCL2 expression was significantly reduced by TAK-242 (Table 3), similar to blocking the NF-kB signal pathway with BAY117082 (Fig. 7). The p38 inhibitor also reduced CXCL8 and CXCL1 chemokines when stimulated with Saliva Orthana ${ }^{\circledR}$. Blocking ERK and JNK pathways via U0126 and SP600125

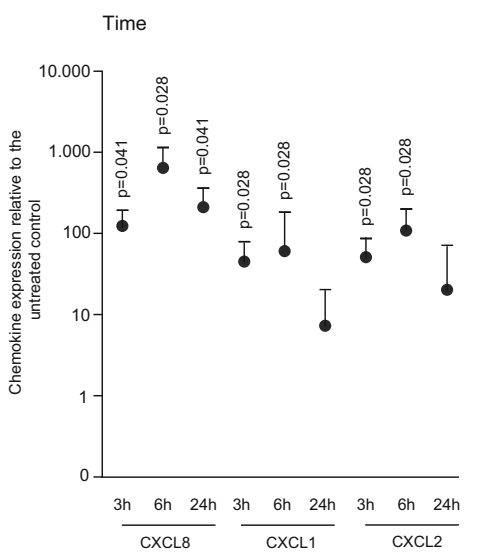

Fig. 5 Time- and dose-dependent mRNA expression of CXCL8, CXCL1, and CXCL2 in response to Saliva Orthana ${ }^{\circledR}$. Gingival fibroblasts were exposed to Saliva Orthana ${ }^{\circledR}$ at indicated time points and with the indicated dilutions. CXCL8 expression was had no impact on chemokine expression (Fig. 7). Saliva Orthana ${ }^{\circledR}$, whole saliva, and mucins from bovine submaxillary glands all enhanced phospho-NF-kB signaling in gingival fibroblasts, indicating that mucin-containing artificial saliva can activate the NF-KB pathway in gingival fibroblasts (Fig. 7).

\section{Discussion}

Despite the global use of artificial saliva in xerostomia patients, a potential cellular response to artificial saliva has not been investigated so far. We show here that Saliva Orthana ${ }^{\circledR}$ provoked a substantial increase of chemokine expression in gingival fibroblasts. The effects could be mimicked with mucins purified from bovine salivary glands and blocked with

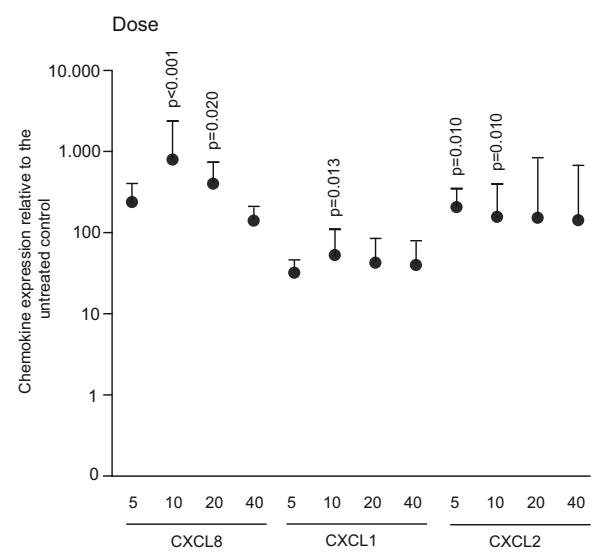

measured with RT-PCR. Data were normalized to expression levels of control cultures with serum-free medium alone. Circles represent the mean \pm standard deviation of three experiments with two cell donors. Not indicated are $p$ values $>0.1$ 
Table 2 Chemokine expression of oral epithelial cells exposed to artificial saliva

\begin{tabular}{lllllllll}
\hline & $\begin{array}{l}\text { Saliva } \\
\text { Orthana }\end{array}$ & SD & Aldiamed & SD & Glandosane & SD & $\begin{array}{c}\text { Saliva } \\
\text { Natura }\end{array}$ & SD \\
\hline CXCL8 & 2.76 & 1.13 & 0.30 & 0.28 & $10.50^{*}$ & 3.71 & 2.04 & 0.63 \\
CXCL1 & 0.52 & 0.18 & 0.23 & 0.15 & 4.69 & 1.09 & 1.06 & 0.36 \\
CXCL2 & 0.52 & 0.24 & 0.31 & 0.25 & 3.89 & 1.11 & 1.13 & 0.35 \\
\hline
\end{tabular}

Data represent the mean \pm standard deviation of three experiments with two cell donors

${ }^{*}$ Indicate $p$ values $<0.05$ inhibitors of TLR4 signaling, implicating a possible involvement of endotoxins. The impact of artificial saliva on the expression of chemokines is by far less pronounced than the broad spectrum response of oral fibroblast to natural saliva [16]. In oral epithelial cells, Glandosane ${ }^{\circledR}$ significantly increased CXCL8 expression. These in vitro data suggest that artificial saliva can modulate chemokine expression with a differential responsiveness of gingival fibroblast and epithelial cells.

Artificial saliva can be distinguished by the containing of mucins, which are linear, heavily O-glycosylated proteins with multiple biological roles [24, 25]. In support of a possible role of mucins, recombinant mucin 1 increased expression of CXCL8 in gingival fibroblasts. Moreover, CXCL8 staining was increased in the epithelium of patients with cystic fibrosis and co-localized with mucins [26]. Mucins are correlated with the severity of periodontal disease [27]. Mucin 2 can even be inversely correlated with CXCL8 expression, at least in pediatric patients with Crohn's disease [28]. Also, splice variants differ in their ability to modulate CXCL8 expression [29].
Thus, mucins in artificial saliva may provoke the expression of CXCL8 in gingival fibroblasts.

TAK-242, the inhibitor of toll-like receptor 4 signaling, blocked chemokine expression in response to Saliva Orthana $^{\circledR}$ and mucins from bovine submaxillary glands. A possible role of endotoxin contamination of natural mucins can therefore not be ruled out. Moreover, NF- $\mathrm{KB}$ and MAP kinase signaling mediate the chemokine expression in response to Saliva Orthana ${ }^{\circledR}$ and mucins. The gene expression pattern by blocking p38, however, is not identical between Saliva Orthana ${ }^{\circledR}$ and purified mucins. On communication lev$\mathrm{el}$, mucins bind to receptors including epidermal growth factor receptor and related subfamilies [30]; yet, blocking of the respective receptor had no impact on the inflammatory response to whole saliva [16]. Thus, the data presented here remain descriptive, leaving room to further discover the underlying molecular mechanism.

Descriptive are also observations with HSC-2 oral epithelial cells. Glandosane ${ }^{\circledR}$ significantly increased CXCL8 expression. The effects of Glandosane ${ }^{\circledR}$ are in line with the
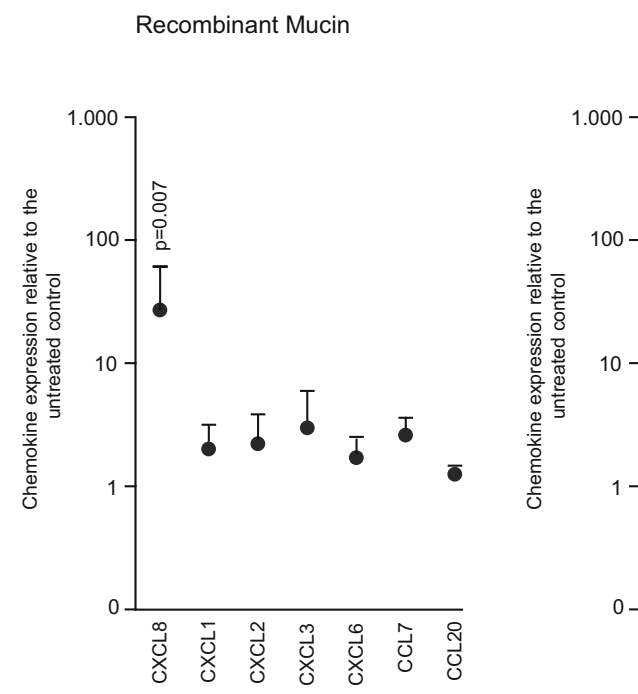

Fig. 6 Chemokine expression of gingival fibroblasts from different mucin origins. Mucins from different origins were utilized to stimulate oral fibroblasts. Cells were stimulated with recombinant human mucin 1 $(25 \mu \mathrm{g} / \mathrm{ml})$, mucins from bovine submaxillary glands $(50 \mu \mathrm{g} / \mathrm{ml})$, and sterile-filtered saliva. After $6 \mathrm{~h}$ of exposure, mRNA was collected and
Bovine Mucin

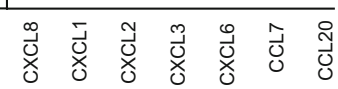

subjected to RT-PCR. Data were normalized to expression levels of control cultures with serum-free medium alone. Circles represent the mean \pm standard deviation of three experiments with two cell donors. Not indicated are $p$ values $>0.1$ 
Table 3 Relative cytokine expression of gingival fibroblasts exposed to Saliva Orthana ${ }^{\circledR}$ and mucin 1

\begin{tabular}{lllllllll}
\hline & Saliva Orthana & SD & $\begin{array}{l}\text { Saliva Orthana } \\
\text { +TAK-242 }\end{array}$ & SD & Mucin 1 & SD & $\begin{array}{l}\text { Mucin 1 } \\
\text { +TAK-242 }\end{array}$ & SD \\
\hline CXCL8 & 154.78 & 188.15 & 0.95 & 1.10 & 217.92 & 302.73 & 0.65 & 0.38 \\
CXCL1 & 15.66 & 16.19 & 0.30 & 0.29 & 34.72 & 46.68 & 0.17 & 0.11 \\
CXCL2 & 19.14 & 15.68 & 1.34 & 1.78 & 81.73 & 113.60 & 0.22 & 0.12 \\
\hline
\end{tabular}

Gingival fibroblasts were stimulated with 10 -fold diluted Saliva Orthana and $50 \mu \mathrm{g} / \mathrm{ml}$ mucin 1, with or without $25 \mu \mathrm{M}$ of TLR4-receptor inhibitor TAK242. Data represent the cytokine expression relative to the untreated control. In all experiments, data were significantly reduced by TAK242. $p<0.05$ effects of carboxymethylcellulose to change CXCL8 expression in $\mathrm{HaCaT}$ keratinocytes [31]. We also observed a consistent trend toward an inhibition of chemokine expression by Aldiamed ${ }^{\circledR}$. Aloe Barbadensis, an ingredient in Aldiamed ${ }^{\circledR}$, has anti-inflammatory activity, as indicated by the decrease of CXCL8 and inflammatory cytokines expression in macrophages [32]. Thus, oral epithelial cells obviously behave differently from gingival fibroblasts in their inflammatory response to artificial saliva. Yet, explanations about the particular role of each component of artificial saliva to cause changes in chemokine expression remain at the level of speculation.

The clinical relevance of the present observation is a matter of speculation. The overall questions are whether or not artificial saliva contributes to oral wound healing and if yes, what the underlying cellular molecular mechanisms are. Theoretically, the increased chemokine expression by gingival fibroblasts attracts cells of the innate immune system that can advance the healing process. In support of this assumption, chronic gastric ulcer healing was significantly impaired in male mucin 2-deficient mice [33]. Moreover, administration of mucin 3-epidermal growth factor-like domains reduced mucosal ulceration in experimental acute colitis [34]. Extensive preclinical research by Bonder et al. supports a role of saliva in wound healing $[35,36]$. Today, however, no preclinical studies are available answering if artificial saliva modulates oral wound healing and if mucins play a role in this context.

Future studies should provide an explanation of how Saliva Orthana ${ }^{\circledR}$ increases chemokine expression, if the response of gingival fibroblasts can be explained by mucins and the possible contamination with endotoxins, and why the response is far less compared to whole saliva. Further research is also possible based on our observations with Saliva Natura ${ }^{\circledR}$ that contains plant mucins from Eriodictyon californicum, but failed to provoke a change in chemokine expression. For epithelial cells, it will be important to understand why Glandosane ${ }^{\circledR}$ increased CXCL8 expression, and what explains Aldiamed ${ }^{\circledR}$ causing a trend toward an inhibition of chemokine expression. The present study clearly provides the scientific basis for future research to better understand possible cellular activity of artificial saliva in its various compositions and target cells. a Saliva Orthana

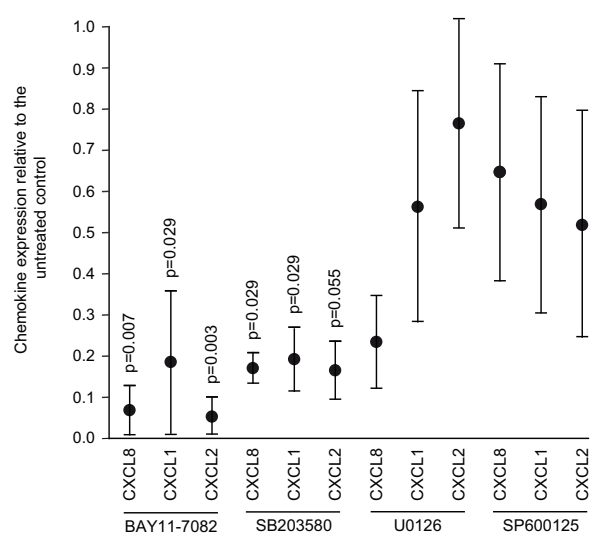

Bovine Mucin

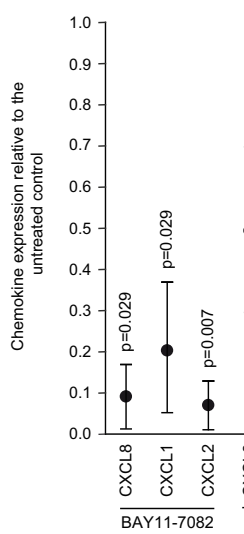

Fig. 7 NF-kB and mitogen-activated protein kinase inhibition and activation of gingival fibroblast chemokine expression. Gingival fibroblasts were exposed to Saliva Orthana ${ }^{\circledR}$ and mucins from bovine submaxillary glands $(50 \mu \mathrm{g} / \mathrm{ml})$. a Signal pathways for NF- $\mathrm{KB}$ and mitogen-activated protein (MAP) kinase were blocked with BAY117082, SB203580, U0126, and SP600125 for NF-kB, p38, ERK, and JNK, respectively. After $6 \mathrm{~h}$ of exposure, mRNA was collected and subjected to RT-PCR. b Saliva Orthana ${ }^{\circledR}$ and bovine mucins increased

b

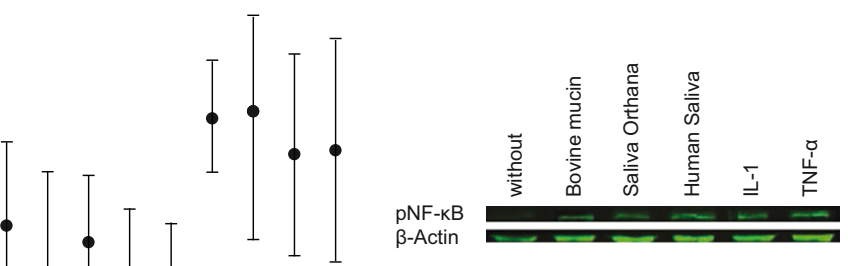

phospho-NF-kB signaling in gingival fibroblasts. Human saliva, 10-fold dilution, interleukin (IL)-1, and tumor necrosis factor (TNF)- $\alpha$, both $10 \mathrm{ng} / \mathrm{ml}$, served as positive, and serum free medium as negative control. Data were normalized to expression levels of control cultures with serum-free medium alone. Circles represent the mean \pm standard deviation of four experiments with two cell donors. Not indicated are $p$ values $>0.1$ 
Acknowledgement We thank Catherine Solioz for her skillful technical assistance. Heinz-Dieter Müller received the Marietta Blau-Fellowship from the Austrian Federal Ministry of Science, Research and Economy (BMWFW-41.922/1, Vienna, Austria).

Conflict of interest The authors declare that they have no competing interests.

\section{References}

1. Humphrey SP, Williamson RT (2001) A review of saliva: normal composition, flow, and function. J Prosthet Dent 85:162

2. Amaechi B, Higham S (2005) Dental erosion: possible approaches to prevention and control. J Dent 33:243

3. Dodds MW, Johnson DA, Yeh C-K (2005) Health benefits of saliva: a review. J Dent 33:223

4. Dawes C (1987) Physiological factors affecting salivary flow rate, oral sugar clearance, and the sensation of dry mouth in man. J Dent Res 66:648

5. Turner M, Jahangiri L, Ship JA (2008) Hyposalivation, xerostomia and the complete denture: a systematic review. J Am Dent Assoc 139:146

6. Lussi A, Jaeggi T (2008) Erosion — diagnosis and risk factors. Clin Oral Investig 12:5

7. Diaz-Arnold AM, Marek CA (2002) The impact of saliva on patient care: a literature review. J Prosthet Dent 88:337

8. Zandim-Barcelos DL, Kielbassa AM, Sampaio JE, Tschoppe P (2015) Saliva substitutes in combination with high-fluoride gel on dentin remineralization. Clin Oral Investig 19:289

9. Aguirre A, Mendoza B, Reddy M, Scannapieco F, Levine M, Hatton M (1989) Lubrication of selected salivary molecules and artificial salivas. Dysphagia 4:95

10. Preetha A, Banerjee R (2005) Comparison of artificial saliva substitutes. Trends Biomater Artif Organs 18:178

11. Aykut-Yetkiner A, Wiegand A, Attin T (2014) The effect of saliva substitutes on enamel erosion in vitro. J Dent 42:720

12. Offner GD, Troxler RF (2000) Heterogeneity of high-molecularweight human salivary mucins. Adv Dent Res 14:69

13. Rousseau K, Kirkham S, Johnson L, Fitzpatrick B, Howard M, Adams EJ, Rogers DF, Knight D, Clegg P, Thornton DJ (2008) Proteomic analysis of polymeric salivary mucins: no evidence for MUC19 in human saliva. Biochem J 413:545

14. Helmerhorst E, Oppenheim F (2007) Saliva: a dynamic proteome. J Dent Res 86:680

15. de Almeida PV, Gregio AM, Machado MA, de Lima AA, Azevedo LR (2008) Saliva composition and functions: a comprehensive review. J Contemp Dent Pract 9:72

16. Cvikl B, Lussi A, Moritz A, Sculean A, Gruber R (2015) Sterilefiltered saliva is a strong inducer of IL- 6 and IL-8 in oral fibroblasts. Clin Oral Investig 19:385

17. Cooper PR, Takahashi Y, Graham LW, Simon S, Imazato S, Smith AJ (2010) Inflammation-regeneration interplay in the dentine-pulp complex. J Dent 38:687

18. Schulze-Osthoff K, Ferrari D, Riehemann K, Wesselborg S (1997) Regulation of NF- $\mathrm{KB}$ activation by MAP kinase cascades. Immunobiology 198:35
19. Werner S, Grose R (2003) Regulation of wound healing by growth factors and cytokines. Physiol Rev 83:835

20. Walton KL, Holt L, Sartor RB (2009) Lipopolysaccharide activates innate immune responses in murine intestinal myofibroblasts through multiple signaling pathways. Am J Physiol Gastrointest Liver Physiol 296:G601

21. Sun J, Ramnath RD, Bhatia M (2007) Neuropeptide substance P upregulates chemokine and chemokine receptor expression in primary mouse neutrophils. Am J Physiol Cell Physiol 293:C696

22. Kawamoto T, Ii M, Kitazaki T, Iizawa Y, Kimura H (2008) TAK242 selectively suppresses Toll-like receptor 4-signaling mediated by the intracellular domain. Eur J Pharmacol 584:40

23. Livak KJ, Schmittgen TD (2001) Analysis of relative gene expression data using real-time quantitative PCR and the $2<\sup >-$ $\Delta \Delta \mathrm{CT}</$ sup $>$ method. Methods 25:402

24. Hattrup CL, Gendler SJ (2008) Structure and function of the cell surface (tethered) mucins. Annu Rev Physiol 70:431

25. McGuckin MA, Lindén SK, Sutton P, Florin TH (2011) Mucin dynamics and enteric pathogens. Nat Rev Microbiol 9:265

26. Burgel PR, Montani D, Danel C, Dusser DJ, Nadel JA (2007) A morphometric study of mucins and small airway plugging in cystic fibrosis. Thorax 62:153

27. Sanchez GA, Miozza VA, Delgado A, Busch L (2013) Relationship between salivary mucin or amylase and the periodontal status. Oral Dis 19:585

28. Hensel KO, Boland V, Postberg J, Zilbauer M, Heuschkel R, Vogel S, Godde D, Wirth S, Jenke AC (2014) Differential expression of mucosal trefoil factors and mucins in pediatric inflammatory bowel diseases. Sci Rep 4:7343

29. Imbert-Fernandez Y, Radde BN, Teng Y, Young WW Jr, Hu C, Klinge CM (2011) MUC1/A and MUC1/B splice variants differentially regulate inflammatory cytokine expression. Exp Eye Res 93: 649

30. Hollingsworth MA, Swanson BJ (2004) Mucins in cancer: protection and control of the cell surface. Nat Rev Cancer 4:45

31. Trier K (2004) In-Vitro-Untersuchung zum Einfluss von Carboxymethylcellulosen auf die Proliferation, die Zytokinliberation und die Bildung freier Sauerstoffradikale humaner Keratinozyten. Med. Dissertation, Friedrich-SchillerUniversität Jena

32. Budai MM, Varga A, Milesz S, Tozser J, Benko S (2013) Aloe vera downregulates LPS-induced inflammatory cytokine production and expression of NLRP3 inflammasome in human macrophages. Mol Immunol 56:471

33. Wallace JL, Vong L, Dharmani P, Srivastava V, Chadee K (2011) Muc-2-deficient mice display a sex-specific, COX-2-related impairment of gastric mucosal repair. Am J Pathol 178:1126

34. Ho SB, Dvorak LA, Moor RE, Jacobson AC, Frey MR, Corredor J, Polk DB, Shekels LL (2006) Gastroenterology. Gastroenterology 131:1501

35. Bodner L, Dayan D, Pinto Y, Hammel I (1993) Characteristics of palatal wound healing in desalivated rats. Arch Oral Biol 38:17

36. Bodner L, Knyszynski A, Adler-Kunin S, Danon D (1991) The effect of selective desalivation on wound healing in mice. Exp Gerontol 26:357 